\title{
Curvas de índices de local em povoamentos de eucalipto obtidas por regressão quantílica
}

\author{
Carlos Alberto Araújo Júnior ${ }^{(1)}$, Carlos Pedro Boechat Soares ${ }^{(1)}$ e Helio Garcia Leite(1) \\ (1)Universidade Federal de Viçosa, Departamento de Engenharia Florestal, Avenida Purdue, s/no, Campus Universitário, Edificio Reinaldo de \\ Jesus Araújo, CEP 36570-900 Viçosa, MG, Brasil. E-mail: araujocaj@gmail.com, csoares@gmail.com, hgleite@gmail.com
}

Resumo - O objetivo deste trabalho foi obter curvas de índice de local, em povoamentos de eucalipto, por meio de regressão quantílica, e compará-las às obtidas com o uso de regressões ajustadas pelo método de mínimos quadrados ordinários. A regressão quantílica pode ser utilizada para a geração de curvas de índice de local, especialmente em presença de dados discrepantes. $\mathrm{O}$ método gera um feixe de curvas mais bem ajustadas aos dados observados, em comparação ao feixe gerado com as estimativas de mínimos quadrados ordinários, seja pelo método da curva-guia (curvas anamórficas), seja pela geração de curvas polimórficas (com o uso de diferentes percentis).

Termos para indexação: Eucalyptus, dados discrepantes, método da curva-guia, mínimos quadrados ordinários, percentis.

\section{Site index curves in eucalyptus plantation obtained by quantile regression}

\begin{abstract}
The objective of this work was to obtain site index curves in eucalyptus plantations by quantile regression, and to compare them to the obtained ones with the use of fitted regressions by the method of ordinary least squares. Quantile regression can be used to generate site index curves, especially in the presence of outliers. The method generates a bundle of curves better adjusted to the observed data, compared to the one generated with the estimates of ordinary least squares, either by the guide curve method (anamorphic curves) or by polymorphic curves (using different percentiles).
\end{abstract}

Index terms: Eucalyptus, outliers, guide curve method, ordinary least squares, percentile.

\section{Introdução}

A classificação da capacidade produtiva das florestas é um dos pilares do manejo florestal (Davis \& Jonhson, 1987). De acordo com Jones (1969) e com Skovsgaard \& Vanclay (2008), essa classificação pode ser feita por meio da avaliação da vegetação indicadora e de elementos do meio ambiente - como clima, solo e outros -, bem como por meio da relação entre a altura das árvores dominantes e a idade dos povoamentos, definindo-se o índice de local.

Como o índice de local fornece uma expressão numérica interpretável, significativamente correlacionada ao crescimento das árvores (Campos \& Leite, 2013), e como os fatores ambientais influenciam o crescimento em altura das árvores dominantes, que é um parâmetro menos afetado pela competição do que outros, como o diâmetro, o índice de local tem sido amplamente utilizado para a classificação da capacidade produtiva de povoamentos de eucalipto (Eucalyptus spp.). Em povoamentos superestocados, não submetidos a desbaste e com taxas de mortalidade insignificantes, a média dos diâmetros das árvores dominantes ou de suas áreas basais ou volumes (Oliveira et al., 2008) pode ser empregada para indicar a capacidade produtiva dos locais de cultivo (Leite et al., 2011). No entanto, a alternativa mais eficiente para estabelecer índices de local é ainda com base na altura.

A geração de curvas de índice de local pode ser feita por meio dos métodos da curva-guia, das equações das diferenças, da predição de parâmetros de Hammer e da atribuição preliminar de índices de local (Clutter et al., 1983; Campos \& Leite, 2013). O método da curva-guia é amplamente utilizado no Brasil para geração de curvas anamórficas, e seu uso pode ser feito com parcelas permanentes ou temporárias. Os demais métodos praticamente não têm sido utilizados, em parte pela alta eficiência do método da curva-guia (Dias et al., 2005; Oliveira et al., 2008), em parte pelo fato de as tendências de crescimento serem consideradas, na maioria das vezes, anamórficas (Binoti et al., 2012). Outro motivo 
para o uso generalizado do método da curva-guia é que os demais requerem o uso de parcelas permanentes, com pelo menos três medições. Isso causa o descarte de muitas parcelas, inclusive das mais importantes, oriundas de plantios recentes (Campos \& Leite, 2013). Para a geração de curvas polimórficas, no entanto, $o$ método mais usual tem sido o da predição de parâmetros (Clutter et al., 1983; Elfving \& Kiviste, 1997).

Independentemente do método utilizado, é necessário que se ajuste uma equação de regressão que, normalmente, é gerada com estimativas obtidas com o método de mínimos quadrados, linear (ordinários) ou não linear (iterativo). Embora o método de mínimos quadrados seja usual, seu emprego pode resultar em estimativas de baixa precisão e enviesadas, na presença de dados discrepantes (Koenker \& Basset, 1978). De acordo com esses autores, a regressão quantílica também pode ser utilizada quando os dados não seguem a distribuição normal. Seus estimadores, além de serem eficientes mesmo com erros que não apresentam distribuição normal, podem ser obtidos com programação matemática ou com programação linear.

Embora a regressão quantílica tenha grande potencial de uso na classificação da capacidade produtiva de povoamentos florestais, pela geração de curvas de índice de local, pouco se sabe sobre sua utilização para este fim. Na pesquisa florestal, ela tem sido empregada em trabalhos sobre a predição da distribuição de diâmetros (Mehtätalo et al., 2008), do crescimento de árvores (Coomes \& Allen, 2007a), da mortalidade (Coomes \& Allen, 2007b), da sucessão ecológica (Dahlgren et al., 2006), do crescimento em altura após incêndio (Meyer et al., 2005), de interação genótipo x ambiente (Vaz et al., 2008) e para o ajuste de funções de afilamento (Cao \& Wang, 2015).

O objetivo deste trabalho foi obter curvas de índice de local, em povoamentos de eucalipto, por meio de regressão quantílica (RQ), e compará-las às obtidas com o uso de regressões ajustadas pelo método de mínimos quadrados ordinários (MQO).

\section{Material e Métodos}

Foram utilizados, inicialmente, dados de oito parcelas permanentes, cada uma com $400 \mathrm{~m}^{2}$, com medições prévias realizadas em quatro ocasiões, nas idades próximas a 24, 36, 48 e 60 meses. Essas parcelas eram provenientes de inventário florestal contínuo do plantio de eucalipto (Eucalyptus urophylla $\mathrm{x}$ Eucalyptus grandis), realizado com espaçamento inicial entre plantas de $3 \times 3 \mathrm{~m}$, na região Norte do Estado de Minas Gerais. Em cada parcela, foram medidas as alturas de cinco árvores dominantes, as quais foram escolhidas seguindo a definição dada por Assman (1970).

A relação entre a altura média das árvores dominantes e as respectivas idades foi definida pelo modelo proposto por Schumacher (1939), utilizado por Demolinari et al. (2007) e por Dias et al. (2005), denominado aqui de modelo 1, conforme $\mathrm{LnHd}=\beta_{0}+\beta_{1}(1 / \mathrm{I})+\varepsilon$, em que: $\mathrm{Hd}$ é a média das alturas totais das 5 árvores dominantes na parcela (m); I é a idade das árvores dominantes, em meses; Ln é o logaritmo neperiano; $\beta_{\mathrm{i}}$, com i igual a $0 \mathrm{e}$ 1, são parâmetros do modelo; e $\varepsilon$ é o erro aleatório, em que $\varepsilon \sim \operatorname{NID}\left(0, \sigma^{2}\right)$.

As estimativas dos parâmetros do modelo 1 foram obtidas pelo método dos mínimos quadrados ordinários (MQO), pela solução do seguinte sistema de equações normais: $\widehat{\beta}=\left(X^{\prime} X\right)^{-1} X^{\prime} Y$, em que $X=1 / I$; e $Y=L n H d$.

Para a regressão quantílica (RQ), as estimativas dos parâmetros do modelo 1 foram obtidas pelo Simplex, com uso da biblioteca quantreg (Koenker, 2013) do programa R (R Development Core Team, 2014):

$$
\operatorname{Min} n^{-1} \sum_{i=1}^{n} \rho_{\theta}\left(Y_{t}-X^{\prime} \beta\right),
$$

em que $\rho$ é uma função de "check", definida por

$$
\rho_{\theta}(u)=\left\{\begin{array}{ll}
q u & u \geq 0 \\
(q-1) u & u<0
\end{array},\right.
$$

tal que: q é um dado percentil, e u é igual ao erro ou resíduo.

Inicialmente, as equações referentes ao modelo 1 foram ajustadas pelos métodos MQO e RQ - com percentil (q) igual a $50 \%$, ou segundo quartil -, sem a presença de dados discrepantes. Em seguida, para verificar o comportamento das estimativas dos parâmetros das equações, na presença de dados discrepantes e em função do método de ajuste (MQO ou RQ), variou-se, arbitrariamente e a intervalos de 4,0 $\mathrm{m}$, a altura média das árvores dominantes de uma das parcelas, aos 24 meses de idade, de modo a torná-la um ponto discrepante da distribuição dos dados. As equações ajustadas com os dados discrepantes foram utilizadas no método da curva-guia, com idade índice de 48 meses, para a geração de curvas 
anamórficas, as quais foram dispostas em gráfico, para mostrar a tendência dos feixes de curvas de índice de local. A significância das estimativas dos parâmetros das equações ajustadas pelos dois métodos foi avaliada pelo teste t, e a precisão das equações, pelo coeficiente de determinação $\left(\mathrm{R}^{2}\right)$ (Kvålseth, 1985). Para avaliar o potencial da RQ na geração de um feixe de curvas de índice de local, sem a presença de dados discrepantes e sem utilizar o método da curva-guia, foram ajustadas equações referentes ao modelo 1 , tendo-se considerado diferentes percentis dos dados observados. Para avaliar a qualidade do ajuste da regressão quantílica em cada percentil da distribuição dos dados, utilizouse a estatística $\mathrm{R}^{1}$, conforme descrita por Koenker \& Machado (1999), dada por

$$
\mathrm{R}^{1}=1-[\hat{\mathrm{V}}(\tau) / \tilde{\mathrm{V}}(\tau)]
$$

em que: $\widehat{V}(\tau)$ é a soma dos erros absolutos ponderados do modelo restrito, que considera apenas o intercepto, e $\tilde{\mathrm{V}}(\tau)$ é a soma dos erros absolutos ponderados do modelo irrestrito, ambos obtidos do processo de obtenção dos parâmetros para os dois métodos (MQO e RQ).

Para avaliar a metodologia proposta neste trabalho, também em uma situação mais próxima da encontrada em uma empresa florestal, considerou-se outro banco de dados de inventário florestal, composto de 261 parcelas permanentes, localizadas em plantios de eucalipto em diferentes condições de produtividade, medidas em diferentes idades (24 a 72 meses).

Novamente, ajustou-se o modelo 1 com os métodos MQO e RQ, e avaliou-se o método da curva-guia, com idade índice de 72 meses, bem como a regressão quantílica associada a diferentes percentis, para geração de curvas de índice de local. A avaliação considerou a análise gráfica dos resultados e as estatísticas referentes ao ajuste das equações em cada método, ou seja: significância dos parâmetros, coeficiente de determinação e estatística $\mathrm{R}^{1}$, conforme descrito anteriormente.

\section{Resultados e Discussão}

As estimativas dos parâmetros das equações ajustadas pelos métodos MQO e RQ, com percentil 50, foram todas significativas (Tabela 1). Como as médias das alturas das árvores dominantes, em todas as idades, foram próximas dos valores de suas respectivas medianas (Tabela 2), os métodos forneceram estimativas de parâmetros bem próximas e, consequentemente, os comportamentos das curvas ajustadas por MQO e RQ também foram semelhantes (Figura 1).

Ao se variar a altura dominante de uma das parcelas, aos 24 meses de idade, de 3,3 para 23,3 m (inclusão de

Tabela 1. Estimativas dos parâmetros e estatísticas das equações ajustadas pelos métodos dos mínimos quadrados ordinários (MQO) e pela regressão quantílica (RQ).

\begin{tabular}{lcccc}
\hline Parâmetro & Estimativa & Erro padrão & Valor $\mathrm{p}$ & $\mathrm{R}^{2}$ \\
\hline \multicolumn{5}{c}{ Mínimos quadrados ordinários } \\
$\beta_{0}$ & 3,5578 & 0,0336 & $<0,01$ & 0,89 \\
$\beta_{1}$ & $-19,9150$ & 1,2443 & $<0,01$ & 0,01 \\
\hline \multicolumn{5}{c}{ Regressão quantílica } \\
$\beta_{0}$ & 3,5546 & 0,0427 & $<0,01$ & 0,89 \\
$\beta_{1}$ & $-19,7945$ & 1,6242 & $<0,01$ & \\
\hline
\end{tabular}

Tabela 2. Média e mediana das alturas das árvores dominantes em cada idade

\begin{tabular}{lcc}
\hline $\begin{array}{l}\text { Idade } \\
\text { (meses) }\end{array}$ & Média de alturas & Mediana de alturas \\
\hline 24 & $-15,8$ & 15,9 \\
36 & 20,6 & 21,0 \\
48 & 23,5 & 23,3 \\
60 & 25,8 & 25,9 \\
\hline
\end{tabular}

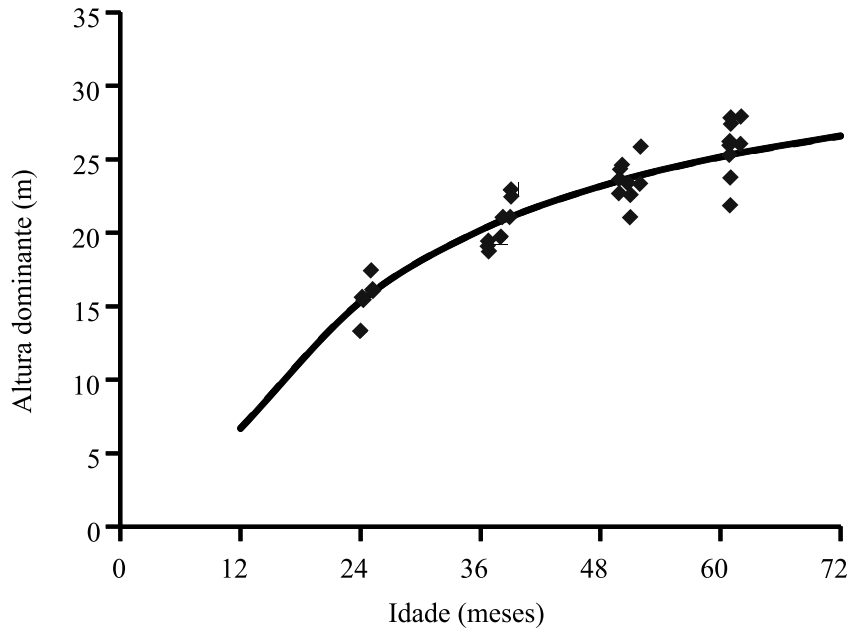

Figura 1. Tendência de crescimento em altura dominante, conforme as equações ajustadas pelo método dos mínimos quadrados ordinários (linha contínua) e pela regressão quantílica (linha tracejada). Os pontos indicam as alturas dominantes observadas em cada parcela e em cada medição. 
dado discrepante), as estimativas dos coeficientes em cada método de ajuste resultaram em menor de precisão das equações, embora todos os parâmetros do modelo tenham permanecido significativos $(\mathrm{p}<0,01)$. As curvas geradas pelas equações de RQ (percentil 50) e MQO apresentam, neste caso, comportamentos distintos (Figura 2). Quanto mais discrepante o ponto, mais distante tornaram-se as curvas, uma vez que a média das alturas dominantes distanciou-se de sua mediana. Neste caso, segundo Chambers \& Tzavidis (2006), a regressão quantílica deve ser preferencialmente utilizada.

Quando se consideraram as alturas dominantes de 3,3 e 7,3 m (Figura 2), situações em que a altura incluída $(\mathrm{Hd}=23 \mathrm{~m})$ constituíam dado mais fortemente discrepante, as curvas resultantes das equações geradas com o método MQO não passaram pela maior concentração de dados. Assim, se os dados da variável resposta estiverem distribuídos assimetricamente, o método MQO tende a fornecer uma curva com desvio da posição central da distribuição, em razão do deslocamento do valor médio. Este fato evidencia a sensibilidade desse método à ocorrência de observações que não obedecem ao padrão linear da maioria dos dados, conforme mencionado por Rousseeuw \& Hubert (2011).
A retirada de valores discrepantes poderia tornar o comportamento das curvas muito parecido (Figura 2). No entanto, a manipulação dos dados requer cuidado, uma vez que um dado discrepante não necessariamente deve ser considerado um erro, mas um comportamento inesperado do fenômeno em estudo, cujas causas devem investigadas antes de sua retirada (Draper \& Smith, 1981). Além disso, por ocasião do processamento de uma grande quantidade de dados de inventário florestal, o processo de eliminação de dados discrepantes pode ser oneroso.

A RQ propiciou melhor distribuição do feixe de curvas anamórficas (curva-guia), na presença de dados discrepantes (Figura 3), quando se utilizaram os coeficientes apresentados na Tabela 3, tanto em idades menores (24 meses) quanto em idades maiores (60 meses). As curvas geradas pelos dois métodos foram coincidentes quanto à idade índice (48 meses), uma vez que, no método da curva-guia, o índice de local é igual à altura média das árvores dominantes nessa idade.

Os dados discrepantes aos 24 meses (Figura 3) tornaram diferentes os comportamentos das curvas de índice de local, nos dois métodos avaliados. As curvas geradas pelas equações ajustadas com MQO podem superestimar ou subestimar a capacidade produtiva
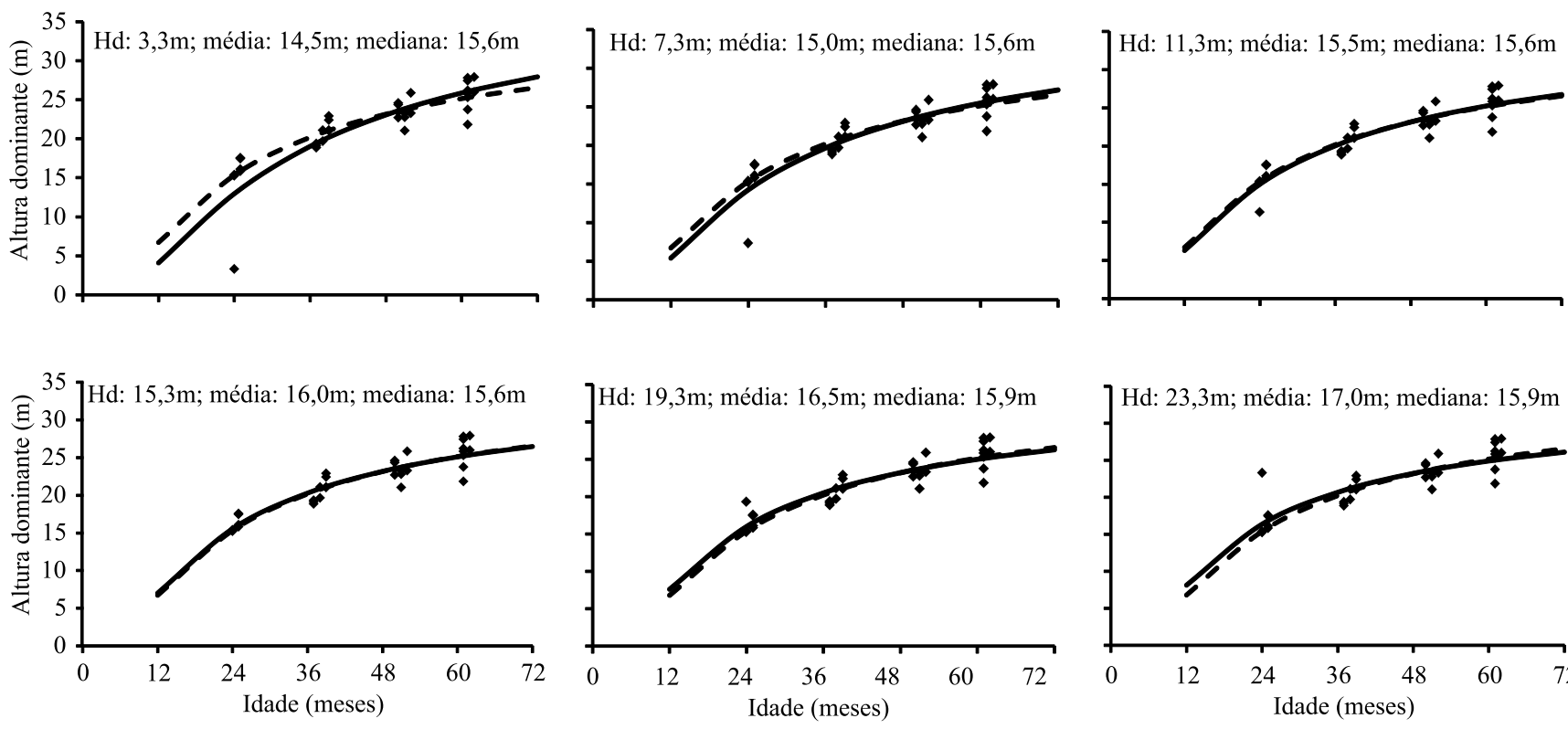

Figura 2. Tendência de crescimento em altura dominante (Hd), conforme as equações ajustadas pelo método dos mínimos quadrados (linhas contínuas) e pela regressão quantílica (linhas tracejadas), com variação da altura dominante, em parcela com 24 meses de idade. Os pontos indicam alturas dominantes observadas em cada parcela e em cada medição.

Pesq. agropec. bras., Brasília, v.51, n.6, p.720-727, jun. 2016 DOI: 10.1590/S0100-204X2016000600003 
de plantios jovens (aos 24 meses), com consequências prejudiciais para a estimativa do estoque volumétrico em idades maiores, o que pode comprometer a tomada de decisão em empreendimentos florestais (Salles et al., 2012).

Ao se ajustar o modelo 1 de acordo com os diferentes percentis, observou-se que as estimativas dos parâmetros também foram estatisticamente significativas, independentemente do percentil utilizado, mas diferentes em cada percentil (Tabela 4). Essa diferença, provavelmente, ocorreu pelo fato de terem sido adotados três percentis na avalição, já que o uso de muitos percentis pode gerar curvas muito similares, até mesmo indistinguíveis graficamente (Bohora \& Cao, 2014).

Como para cada percentil existe um conjunto de parâmetros distintos (Tabela 4), foi possível a obtenção de um feixe de curvas polimórficas com o
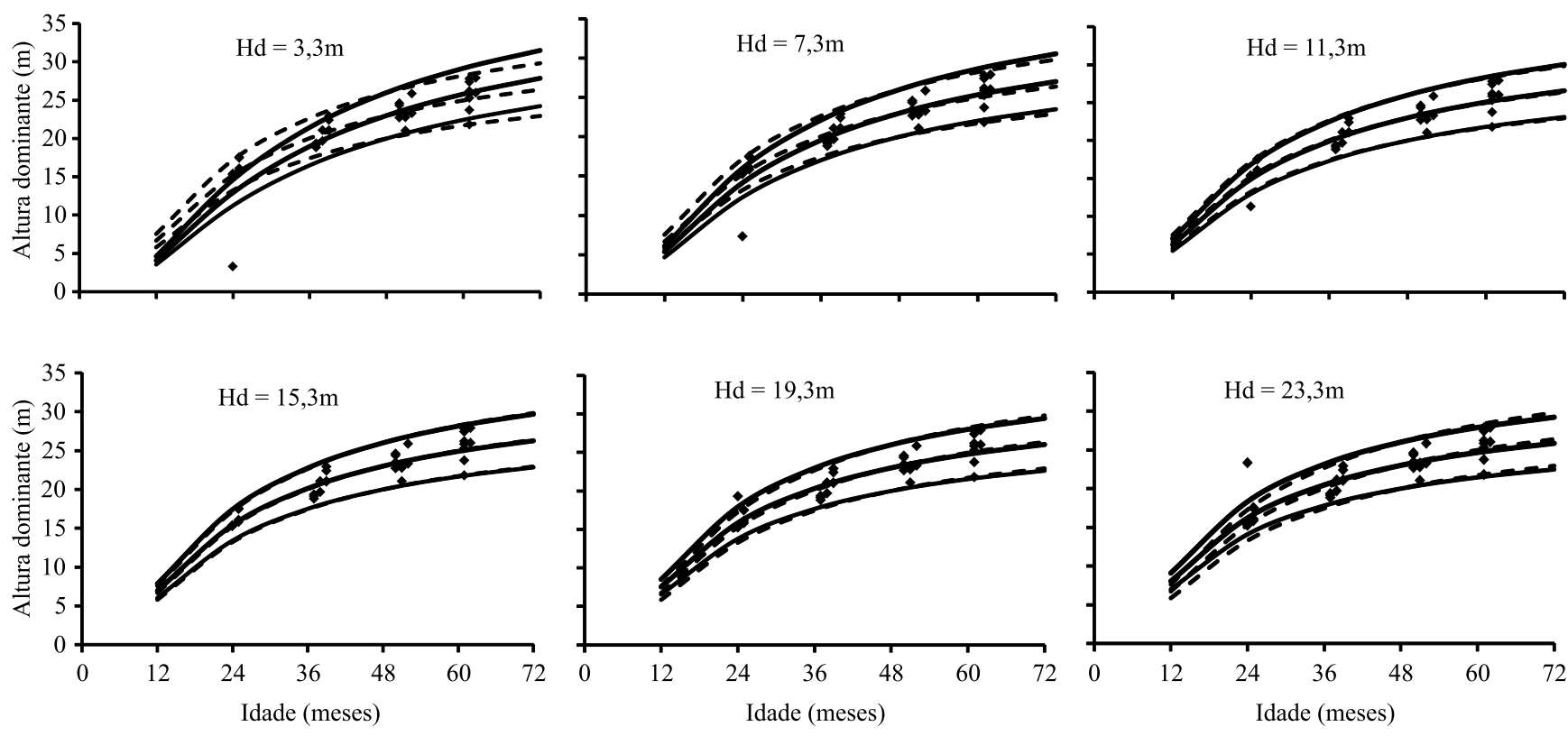

Figura 3. Curvas de índice de local, obtidas pelo método dos mínimos quadrados (linhas contínuas) e regressão quantílica (linhas tracejadas), com variação da altura dominante (Hd) em parcela com 24 meses. Os pontos indicam alturas dominantes observadas em cada parcela e em cada medição.

Tabela 3. Estimativas dos parâmetros e estatísticas das equações ajustadas pelos métodos dos mínimos quadrados ordinários (MQO) e pela regressão quantílica (RQ), com a variação da média da altura dominante (Hd) de uma parcela, aos 24 meses.

\begin{tabular}{|c|c|c|c|c|c|c|c|c|c|c|c|c|}
\hline Parâmetro & Estimativa & Erro padrão & Valor $\mathrm{p}$ & $\mathrm{R}^{2}$ & Estimativa & Erro padrão & Valor $\mathrm{p}$ & $\mathrm{R}^{2}$ & Estimativa & Erro padrão & Valor $\mathrm{p}$ & $\mathrm{R}^{2}$ \\
\hline & \multicolumn{3}{|c|}{$\mathrm{MQO}, \mathrm{Hd}=3,3 \mathrm{~m}$} & & \multicolumn{3}{|c|}{$\mathrm{MQO}, \mathrm{Hd}=7,3 \mathrm{~m}$} & \multicolumn{5}{|c|}{$\mathrm{MQO}, \mathrm{Hd}=11,3 \mathrm{~m}$} \\
\hline$\beta_{0}$ & 3,7138 & 0,1297 & $<0,01$ & \multirow{2}{*}{0,50} & 3,6249 & 0,0689 & $<0,01$ & \multirow{2}{*}{0,71} & 3,5760 & 0,0405 & $<0,01$ & \multirow{2}{*}{0,85} \\
\hline$\beta_{1}$ & $-27,6400$ & 4,8101 & $<0,01$ & & $-23,2400$ & 2,5548 & $<0,01$ & & $-20,8180$ & 1,5021 & $<0,01$ & \\
\hline & \multicolumn{3}{|c|}{$\mathrm{RQ}, \mathrm{Hd}=3,3 \mathrm{~m}$} & & \multicolumn{3}{|c|}{$\mathrm{RQ}, \mathrm{Hd}=7,3 \mathrm{~m}$} & \multicolumn{5}{|c|}{$\mathrm{RQ}, \mathrm{Hd}=11,3 \mathrm{~m}$} \\
\hline$\beta_{0}$ & 3,5546 & 0,0347 & $<0,01$ & \multirow{2}{*}{0,45} & 3,5546 & 0,0376 & $<0,01$ & \multirow{2}{*}{0,69} & 3,5546 & 0,0472 & $<0,01$ & \multirow{2}{*}{0,85} \\
\hline$\underline{\beta_{1}}$ & $-19,7950$ & 1,0945 & $<0,01$ & & $-19,7950$ & 1,3660 & $<0,01$ & & $-19,7950$ & 1,8376 & $<0,01$ & \\
\hline & \multicolumn{3}{|c|}{$\mathrm{MQO}, \mathrm{Hd}=15,3 \mathrm{~m}$} & & \multicolumn{3}{|c|}{$\mathrm{MQO}, \mathrm{Hd}=19,3 \mathrm{~m}$} & \multicolumn{5}{|c|}{$\mathrm{MQO}, \mathrm{Hd}=23,3 \mathrm{~m}$} \\
\hline$\beta_{0}$ & 3,5421 & 0,0310 & $<0,01$ & \multirow{2}{*}{0,89} & 3,5161 & 0,0355 & $<0,01$ & \multirow{2}{*}{0,85} & 3,4950 & 0,0450 & $<0,01$ & \multirow{2}{*}{0,76} \\
\hline$\beta_{1}$ & $-19,1390$ & 1,1508 & $<0,01$ & & $-17,8520$ & 1,3175 & $<0,01$ & & $-16,8080$ & 1,6694 & $<0,01$ & \\
\hline & \multicolumn{3}{|c|}{$\mathrm{RQ}, \mathrm{Hd}=15,3 \mathrm{~m}$} & & \multicolumn{3}{|c|}{$\mathrm{RQ}, \mathrm{Hd}=19,3 \mathrm{~m}$} & \multicolumn{5}{|c|}{$\mathrm{RQ}, \mathrm{Hd}=23,3 \mathrm{~m}$} \\
\hline$\beta_{0}$ & 3,5546 & 0,0368 & $<0,01$ & \multirow{2}{*}{0,89} & 3,5161 & 0,0355 & $<0,01$ & \multirow{2}{*}{0,84} & 3,5521 & 0,0426 & $<0,01$ & \multirow{2}{*}{0,73} \\
\hline$\beta_{1}$ & $-19,7950$ & 1,0775 & $<0,01$ & & $-17,8520$ & 1,3175 & $<0,01$ & & $-19,6400$ & 1,5575 & $<0,01$ & \\
\hline
\end{tabular}


uso da RQ (Figura 4 A), diferentemente do uso do método da curva-guia, que gera um feixe de curvas anamórficas. As curvas geradas, no entanto, não apresentam comportamento muito distinto, em razão da proximidade das estimativas dos parâmetros das equações ajustadas, nos diferentes percentis. No entanto, ao se considerar uma base de dados mais ampla e com maior variabilidade dos valores de altura dominante em cada época de medição, espera-se que as curvas apresentem comportamentos mais distintos.

Outro aspecto importante foi que a geração das curvas de índice de local com uso da RQ, nos diferentes percentis, independe de uma idade índice selecionada arbitrariamente, como no método da curva-guia. Além disso, é possível classificar em qual percentil se encontra determinado povoamento na distribuição dos dados e, neste caso, o próprio percentil pode ser considerado um índice de local, em que os povoamentos classificados nos maiores percentis são os de maior capacidade produtiva (Lekwadi et al., 2012). Binoti et al. (2012) destacam que o método da curva-guia, por gerar curvas anamórficas, pode não captar tendências distintas de crescimento, o que destaca os resultados obtidos com o uso de percentis.

Levando-se em conta o banco de dados de inventário com 261 parcelas permanentes, observou-se que as equações ajustadas apresentaram coeficientes de determinação próximos; no entanto, as estimativas

Tabela 4. Estimativas dos parâmetros e estatísticas das equações ajustadas pela regressão quantílica (RQ), para diferentes percentis $\left(\mathrm{P}_{1}, \mathrm{P}_{30}, \mathrm{P}_{50}, \mathrm{P}_{70}, \mathrm{P}_{99}\right)$.

\begin{tabular}{lcccc}
\hline Parâmetro & Estimativa & Erro padrão & Valor $\mathrm{p}$ & $\mathrm{R}^{2}$ \\
\hline \multicolumn{5}{c}{ Percentil $\mathrm{P}_{1}$} \\
$\beta_{0}$ & 3,4023 & 0,0821 & $<0,01$ \\
$\beta_{1}$ & $-19,5336$ & 3,3427 & $<0,01$ & 0,69 \\
\hline \multicolumn{5}{c}{ Percentil $\mathrm{P}_{30}$} \\
$\beta_{0}$ & 3,4879 & 0,0593 & $<0,01$ \\
$\beta_{1}$ & $-18,3225$ & 2,5021 & $<0,01$ & 0,69 \\
\hline \multicolumn{5}{c}{ Percentil $\mathrm{P}_{50}$} \\
$\beta_{0}$ & 3,5546 & 0,0400 & $<0,01$ \\
$\beta_{1}$ & $-19,7944$ & 1,4300 & $<0,01$ \\
\hline \multicolumn{5}{c}{ Percentil $\mathrm{P}_{70}$} \\
$\beta_{0}$ & 3,6066 & 0,0514 & $<0,01$ \\
$\beta_{1}$ & $-20,7250$ & 2,0677 & $<0,01$ \\
\hline \multicolumn{5}{c}{ Percentil $\mathrm{P}_{99}$} \\
$\beta_{0}$ & 3,6517 & 0,0222 & $<0,01$ \\
$\beta_{1}$ & $-19,6822$ & 1,0550 & $<0,01$ \\
\hline
\end{tabular}
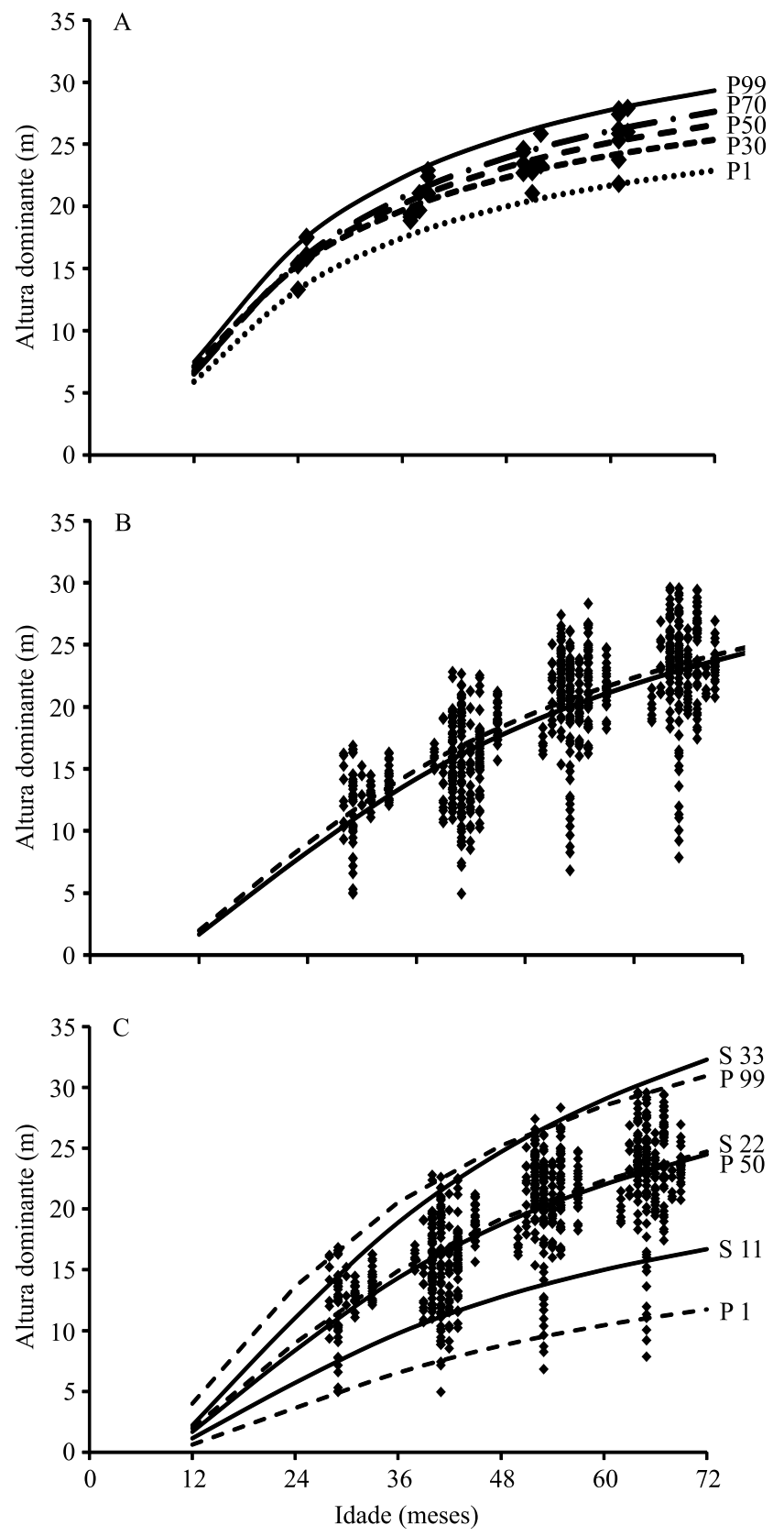

Figura 4. Curvas de índice de local, obtidas conforme as equações ajustadas pela regressão quantílica para diferentes percentis - $\mathrm{P}_{1}, \mathrm{P}_{30}, \mathrm{P}_{50}, \mathrm{P}_{70}, \mathrm{P}_{99}-(\mathrm{A})$. Tendência de crescimento em altura dominante, a partir das equações ajustadas pelo método dos mínimos quadrados ordinários (linha contínua) e pela regressão quantílica (linha tracejada), para o banco de dados com 261 parcelas permanentes (B). Curvas de índice de local obtidas pelo método dos mínimos quadrados ordinários (linhas contínuas) e regressão quantílica (linhas tracejadas), para o banco de dados com 261 parcelas permanentes (C). Os pontos indicam as alturas dominantes observadas em cada parcela e em cada medição. 
dos coeficiente angulares $\left(\beta_{1}\right)$ foram discrepantes (Tabela 5). Os menores valores para $\beta_{1}$ indicam que as estimativas geradas pelo método de MQO são menores do que as obtidas pela aplicação da RQ (Figura 4 B).

Quando se consideraram as equações ajustadas para os diferentes percentis conforme a regressão quantílica (Tabela 6), bem como as estimativas dos parâmetros da equação ajustada pelo MQO (Tabela 5), associada ao método da curva-guia, observou-se que os métodos geraram curvas de índice de local com comportamentos distintos: polimórficas para a RQ e anamórficas para o MQO (Figura $4 \mathrm{C}$ ). Além disso, para as curvas indicativas de maior capacidade produtiva, a regressão quantílica (RQ) apresentou melhor concordância entre os valores estimados e os observados, ao passo que a curva gerada pelo MQO aparentemente superestimou esses valores.

Tabela 5. Estimativas dos parâmetros e estatísticas das equações ajustadas pelos métodos dos mínimos quadrados ordinários (MQO) e pela regressão quantílica (RQ), para o banco de dados com 261 parcelas permanentes.

\begin{tabular}{|c|c|c|c|c|}
\hline Parâmetro & Estimativa & Erro padrão & Valor $\mathrm{p}$ & $\mathrm{R}^{2}$ \\
\hline & \multicolumn{4}{|c|}{ MQO } \\
\hline$\beta_{0}$ & 3,72600 & 0,02324 & $<0,01$ & \multirow{2}{*}{0,56} \\
\hline \multirow[t]{2}{*}{$\beta_{1}$} & $-38,60489$ & 1,12417 & $<0,01$ & \\
\hline & \multicolumn{4}{|c|}{ RQ } \\
\hline$\beta_{0}$ & 3,71312 & 0,02180 & $<0,01$ & \multirow{2}{*}{0,54} \\
\hline$\underline{\beta_{1}}$ & $-36,39032$ & 1,16039 & $<0,01$ & \\
\hline
\end{tabular}

Tabela 6. Estimativas dos parâmetros e estatísticas das equações ajustadas pela regressão quantílica (RQ), para diferentes percentis $\left(\mathrm{P}_{1}, \mathrm{P}_{50}, \mathrm{P}_{99}\right)$, para o banco de dados com 261 parcelas permanentes.

\begin{tabular}{lrrrr}
\hline Parâmetro & Estimativa & Erro padrão & Valor $\mathrm{p}$ & $\mathrm{R}^{2}$ \\
\hline \multicolumn{5}{c}{ Percentil $\mathrm{P}_{1}$} \\
$\beta_{0}$ & 3,04932 & 0,27756 & $<0,01$ & \\
$\beta_{1}$ & $-42,19474$ & 12,81139 & $<0,05$ & 0,19 \\
\hline \multicolumn{5}{c}{ Percentil $\mathrm{P}_{50}$} \\
$\beta_{0}$ & 3,71312 & 0,02180 & $<0,01$ & 0,40 \\
$\beta_{1}$ & $-36,39032$ & 1,16039 & $<0,01$ & 0,40 \\
\hline \multicolumn{5}{c}{ Percentil $\mathrm{P}_{99}$} \\
$\beta_{0}$ & 3,84267 & 0,02003 & $<0,01$ & 0 \\
$\beta_{1}$ & $-29,53124$ & 0,75560 & $<0,01$ & 0,37 \\
\hline
\end{tabular}

\section{Conclusões}

1. A regressão quantílica é adequada para a geração de curvas de índice de local em povoamentos de eucalipto.

2. A regressão quantílica gera curvas polimórficas (com uso de diferentes percentis) ou anamórficas (com o uso do método da curva-guia), o que comprova sua flexibilidade na classificação da capacidade produtiva de povoamentos florestais.

3. As estimativas realizadas com a regressão quantílica, com o uso de diferentes percentis, geram um feixe de curvas mais bem ajustadas entre os dados observados do que o feixe gerado pelo método da curva-guia, com uso das estimativas de mínimos quadrados, especialmente em presença de dados discrepantes.

\section{Referências}

ASSMAN, E. The principles of forest yield study. Oxford: Pergamon Press, 1970. 506p.

BINOTI, D.H.B.; BINOTI, M.L.M. da S.; LEITE, H.G. Aplicação da função hiperbólica na construção de curvas de índice de local. Revista Árvore, v.36, p.741-746, 2012. DOI: 10.1590/ S0100-67622012000400015.

BOHORA, S.B.; CAO, Q.V. Prediction of tree diameter growth using quantile regression and mixed-effects models. Forest Ecology and Management, v.319, p.62-66, 2014. DOI: 10.1016/j. foreco.2014.02.006.

CAMPOS, J.C.C.; LEITE, H.G. Mensuração Florestal: perguntas e respostas. 4. ed. Viçosa: Ed. da UFV, 2013. 600p.

CAO, Q.V.; WANG, J. Evaluation of methods for calibrating a tree taper equation. Forest Science, v.61, p.213-219, 2015. DOI: 10.5849/forsci.14-008.

CHAMBERS, R.; TZAVIDIS, N. $M$-quantile models for small area estimation. Biometrika, v.93, p.255-268, 2006. DOI: 10.1093/ biomet/93.2.255.

CLUTTER, J.L.; FORTSON, J.C.; PIENAAR, L.V.; BRISTER, G.H.; BAILEY, R.L. Timber management: a quantitative approach. $3^{\text {th }}$ ed. New York: J. Willey, 1983. 333p.

COOMES, D.A.; ALLEN, R.B. Effects of size, competition and altitude on tree growth. Journal of Ecology, v.95, p.1084-1097, 2007a. DOI: 10.1111/j.1365-2745.2007.01280.x.

COOMES, D.A.; ALLEN, R.B. Mortality and tree-size distributions in natural mixed-age forests. Journal of Ecology, v.95, p.27-40, 2007b. DOI: 10.1111/j.1365-2745.2006.01179.x.

DAHLGREN, J.P.; ERIKSSON, O.; BOLMGREN, K.; STRINDELL, M.; EHRLÉN, J. Specific leaf area as a superior predictor of changes in field layer abundance during forest succession. Journal of Vegetation Science, v. 17, p.577-582, 2006. DOI: $10.1111 / j .1654-1103.2006 . t b 02481 . x$. 
DAVIS, L.S.; JOHNSON, K.N. Forest management. $3^{\text {th }}$ ed. New York: McGraw-Hill, 1987. 790p.

DEMOLINARI, R. de A.; SOARES, C.P.B.; LEITE, H.G.; SOUZA, A.L. de. Crescimento de plantios clonais de eucalipto não desbastados na região de Monte Dourado (PA). Revista Árvore, v.31, p.503-512, 2007. DOI: 10.1590/S0100-67622007000300016.

DIAS, A.N.; LEITE, H.G.; NOGUEIRA, G.S.; RODRIGUES, F.L. Avaliação de métodos de ajuste de curvas de índices de local em povoamentos de eucalipto desbastados. Revista Árvore, v.29, p.741-747, 2005. DOI: 10.1590/S0100-67622005000500009.

DRAPER, N.R.; SMITH, H. Applied regression analysis. $2^{\text {nd }}$ ed. New York: J. Willey, 1981. 709p.

ELFVING, B.; KIVISTE, A. Construction of site index equations for Pinus sylvestris L. using permanent plot data in Sweden. Forest Ecology and Management, v.98, p.125-134, 1997. DOI: 10.1016/ S0378-1127(97)00077-7.

JONES, J.R. Review and comparison of site evaluation methods. Fort Collins: Rocky Mountain Forest and Range Experiment Station, 1969. (USDA Forest Service Research Paper RM-51).

KOENKER, R. quantreg: Quantile regression. R package version 5.05. 2013. Disponível em: <http://CRAN.R-project.org/ package $=$ quantreg $>$. Acesso em: 21 nov. 2015 .

KOENKER, R.; BASSETT JR., G. Regression quantiles. Econometrica, v.46, p.33-50, 1978. DOI: 10.2307/1913643.

KOENKER, R.; MACHADO, J.A.F. Goodness of fit and related inference processes for quantile regression. Journal of the American Statistical Association, v.94, p.1296-1310, 1999. DOI: 10.1080/01621459.1999.10473882.

KVÅLSETH, T.O. Cautionary note about $\mathrm{R}^{2}$. The American Statistician, v.39, p.279-285, 1985. DOI: 10.1080/00031305.1985.10479448.

LEITE, H.; CASTRO, R..; SILVA, A.; JÚNIOR, C.; BINOTI, D.; CASTRO, A.F.; BINOTI, M. Classificação da capacidade produtiva de povoamentos de eucalipto utilizando diâmetro dominante. Silva Lusitana, v.19, p.181-195, 2011.

LEKWADI, S.O.; NEMESOVA, A.; LYNCH, T.; PHILLIPS, H.; HUNTER, A.; MAC SIURTAIN, M. Site classification and growth models for Sitka spruce plantations in Ireland. Forest
Ecology and Management, v.283, p.56-65, 2012. DOI: 10.1016/j. foreco.2012.07.003.

MEHTÄTALO, L.; GREGOIRE, T.G.; BURKHART, H.E. Comparing strategies for modeling tree diameter percentiles from remeasured plots. Environmetrics, v.19, p.529-548, 2008. DOI: 10.1002/env.896.

MEYER, K.M.; WARD, D.; MOUSTAKAS, A.; WIEGAND, K. Big is not better: small Acacia mellifera shrubs are more vital after fire. African Journal of Ecology, v.43, p.131-136, 2005. DOI: 10.1111/j.1365-2028.2005.00559.x.

OLIVEIRA, M.L.R. de; LEITE, H.G.; NOGUEIRA, G.S.; GARCIA, S.L.R.; SOUZA, A.L. de. Classificação da capacidade produtiva de povoamentos não desbastados de clones de eucalipto. Pesquisa Agropecuária Brasileira, v.43, p.1559-1567, 2008. DOI: 10.1590/S0100-204X2008001100015.

R CORE TEAM. R: a language and environment for statistical computing. Vienna: R Foundation for Statistical Computing, 2014. Disponível em: <http://www.R-project.org > Acesso em: 21 nov. 2015.

ROUSSEEUW, P.J.; HUBERT, M. Robust statistics for outlier detection. WIREs Data Mining and Knowledge Discovery, v.1, p.73-79, 2011. DOI: 10.1002/widm.2.

SALLES, T.T.; LEITE, H.G.; OLIVEIRA NETO, S.N. de; SOARES, C.P.B.; PAIVA, H.N. de; SANTOS, F.L. dos. Modelo de Clutter na modelagem do crescimento e produção de eucalipto em sistemas de integração lavoura-pecuária-floresta. Pesquisa Agropecuária Brasileira, v.47, p.253-260, 2012. DOI: 10.1590/ S0100-204X2012000200014.

SCHUMACHER, F.X. A new growth curve and its application to timber studies. Journal of Forestry, v.37, p.819-820, 1939.

SKOVSGAARD, J.P.; VANCLAY, J.K. Forest site productivity: a review of the evolution of dendrometric concepts for even-aged stands. Forestry, v.81, p.13-31, 2008. DOI: 10.1093/forestry/ cpm041.

VAZ, S.; MARTIN, C.S.; EASTWOOD, P.D.; ERNANDE, B.; CARPENTIER, A.; MEADEN, G.J.; COPPIN, F. Modelling species distributions using regression quantiles. Journal of Applied Ecology, v.45, p.204-217, 2008. DOI: 10.1111/j.1365-2 664.2007.01392.x.

Recebido em 28 de maio de 2015 e aprovado em 11 de abril de 2016

Pesq. agropec. bras., Brasília, v.51, n.6, p.720-727, jun. 2016 DOI: 10.1590/S0100-204X2016000600003 